\title{
Self-assembled nanomaterials for nonlinear fiber optics and tunable plasmonics
}

Islam Ashry $^{\mathrm{a}}$, Chalongrat Daengngam ${ }^{\mathrm{b}}$, Ishac Kandas ${ }^{\mathrm{a}}$, James Heflin ${ }^{\mathrm{b}}$, Hans Robinson ${ }^{\mathrm{b}}$, Yong Xu* ${ }^{\mathrm{a}}$ ${ }^{a}$ Department of Electrical and Computer Engineering, Virginia Tech, Blacksburg, VA, USA 24061; ${ }^{b}$ Department of Physics, Virginia Tech, Blacksburg, VA, USA 24061

\begin{abstract}
As an amorphous material with full inversion symmetry, silica-based microstructures cannot possess significant secondorder nonlinearity. We recently developed a method that can potentially overcome this deficiency by coating a silica fiber taper with layers of radially aligned nonlinear molecules. The coating process can be accomplished through layerby-layer self-assembly, where the alignment of the nonlinear molecules is maintained through electrostatic interaction. As a result, the nonlinear fiber structures are thermodynamically stable and can generate significant second-order nonlinear responses despite their full rotational symmetry. This prediction has been experimentally confirmed through SHG measurements. To further enhance the overall second-order nonlinearity, we have developed an UV-ablation-based approach that can generate second-order nonlinearity that is spatially periodic along the fiber taper. Our preliminary experiments suggest that SHG intensity can be enhanced by such quasi-phase-matching configurations.

We can also use the self-assembly approach to construct tunable plasmonic systems. As a proof-of-concept study, we assembled swellable polymer films over a planar Au substrate through layer-by-layer assembly and covered the swellable polymer with a monolayer of quantum dots. After immersing the swellable plasmonic structure in solution and adjusting its $\mathrm{pH}$ value, we used a fluorescence lifetime based approach to demonstrate that the thickness of the swellable polymers can be modified by almost $400 \%$. The fluorescence lifetime measurements also confirmed that the plasmonic resonance can be significantly modified by the swellable polymers.
\end{abstract}

Keywords: nonlinear optics, plasmonics, layer-by-layer self-assembly, fiber optics, near field optics

\section{INTRODUCTION}

Ever since their first discovery ${ }^{1}$, layer-by-layer (LbL) self-assembled films have become an important class of nanomaterials that offers significant advantages. First of all, the LbL self-assembly approach allows detailed structural and thickness control at the molecular level. In particular, since the assembly process is based on the electrostatic attraction of interlayer charges, each assembled layer is typically limited to a single monolayer of polymers with thickness in the range of a few nanometers or less. Secondly, the self-assembled films are highly smooth and uniform, which is an important feature for photonics applications. Thirdly, the self-assembly approach relies on electrostatic interaction and is therefore compatible with a wide range of nanomaterials including nonlinear molecules, quantum dots (QDs), and plasmonic nanoparticles (NPs). By selecting appropriate nanomaterials for self-assembly, we can incorporate a large number of functionalities (e.g., second-order nonlinearity and plasmonic resonances) onto common photonic structures such as silica fibers and microspheres. Fourthly, post-fabrication tuning of the self-assembled structures is also possible if we use swellable polymers ${ }^{2}$. Finally, the self-assembly process is simple, straightforward, low cost, and can be carried out without using any specialized equipment or clean room facilities. In the present paper, we focus on two types of functionalities that are difficulty to achieve in traditional optoelectronic devices: silica-based fiber structures with second-order nonlinearity, and tunable plasmonic nanostructures.

It is well known that most silica-based structures and devices cannot support second-order nonlinearity ${ }^{3}$. Fundamentally, this limitation can be attributed to a simple fact: As an amorphous material, silica glass possesses inversion symmetry and cannot possess second-order nonlinearity. Consequently, until now it has been very difficult to use silica fiber to realize important second-order processes including second harmonic generation (SHG) and optical parametric oscillation (OPO). In order to incorporate second-order nonlinearity into silica-based devices, most of the current approaches utilize external field (i.e., poling) to generate a preferred direction in silica glass ${ }^{4-8}$. The poled silica glass, however, is thermodynamically unstable and the resultant second order nonlinearity tends to decay over time 9

* yong@vt.edu; phone: 540-231-2464; fax: 540-231-3362

Ultrafast Imaging and Spectroscopy, edited by Zhiwen Liu, Proc. of SPIE Vol. 8845 , 88450D · @ 2013 SPIE · CCC code: 0277-786X/13/\$18 · doi: 10.1117/12.2024380 
In contrast, our approach ${ }^{10-12}$ relies on coating a cylindrical silica fiber taper with layers of self-assembled nonlinear materials, which has been experimentally shown to be thermodynamically stable ${ }^{13}$. In the present publication, we report the fabrication of such silica-based nonlinear fibers and experimental characterizations of their second-order nonlinearity. We also present some preliminary results showing the feasibility of quasi-phase-matching (QPM) based on UV ablation.

Next, we describe the characterization of a tunable plasmonic structure based on swellable polymers self-assembled over an $\mathrm{Au}$ substrate. A number of different polymer systems are known to change their conformation in response to an external stimulus such as temperature or $\mathrm{pH}$, and are finding an increasing number of applications in diverse fields such as drug delivery, tissue engineering, biosensors, and reconfigurable coatings ${ }^{2}, 14$. In the area of plasmonics, these materials, in thin film form, have been used to modify the plasmon resonances in a few simple geometries. In particular, the case of a metal nanoparticle (NP) suspended by a swellable polymer film above a flat metal surface has been investigated $^{15}$. Here we present a fluorescence lifetime based approach that enables us to characterize the swelling / deswelling of a tunable plasmonic nanostructure.

\section{FABRICATION AND CHARACTERIZATION OF SILICA-BASED SECOND-ORDER NONLINEAR FIBER}

Fabrication of the nonlinear fibers can be divided into two steps. The first step is the fabrication of a silica fiber taper with waist diameter in the range of a few microns. Our taper pulling process is similar to the procedures described in Ref. [16]. Briefly, we placed a multimode silica fiber $(50 \mu \mathrm{m}$ core and $125 \mu \mathrm{m}$ cladding, Corning ClearCurve OM2) between two fiber clamps and heated the fiber using a propane flame. As the glass softens, we slowly pulled the two clamps apart using two motion stages, with pulling speed controlled by a computer.

The next fabrication step involves the LbL assembly of appropriate nonlinear molecules. The assembly process is based on the procedure described in Ref. [13]. Briefly, we dipped the fiber taper into a polycationic solution containing poly(allyamine hydrochloride) (PAH) with $\mathrm{pH} 7.0$ and $10 \mathrm{mM}$ concentration. Through electrostatic interaction, the negatively charged taper surface was uniformly coated with a monolayer of positively charged PAH film. Following PAH deposition, we dipped the fiber sample into an aqueous solution containing nonlinear molecules Procion Brown MX-GRN (PB) at $\mathrm{pH} 10.5,5 \mathrm{mg} / \mathrm{mL}$ in concentration and with $0.5 \mathrm{M} \mathrm{NaCl}$ added. Under this condition, the dichlorotriazine moieties of $\mathrm{PB}$ form covalent bonds with the unprotonated amines of PAH. Upon deposition of the sequential PAH layer at $\mathrm{pH} 7.0$, the negatively-charged sulfonates of $\mathrm{PB}$ bind electrostatically with the protonated amine groups of PAH. The deposition of each monolayer is fast $(<2$ minutes $)$ and can be repeated as many times as desired. A scanning electron microscope (SEM) image of a nonlinear fiber taper is shown in Fig. 1(a).
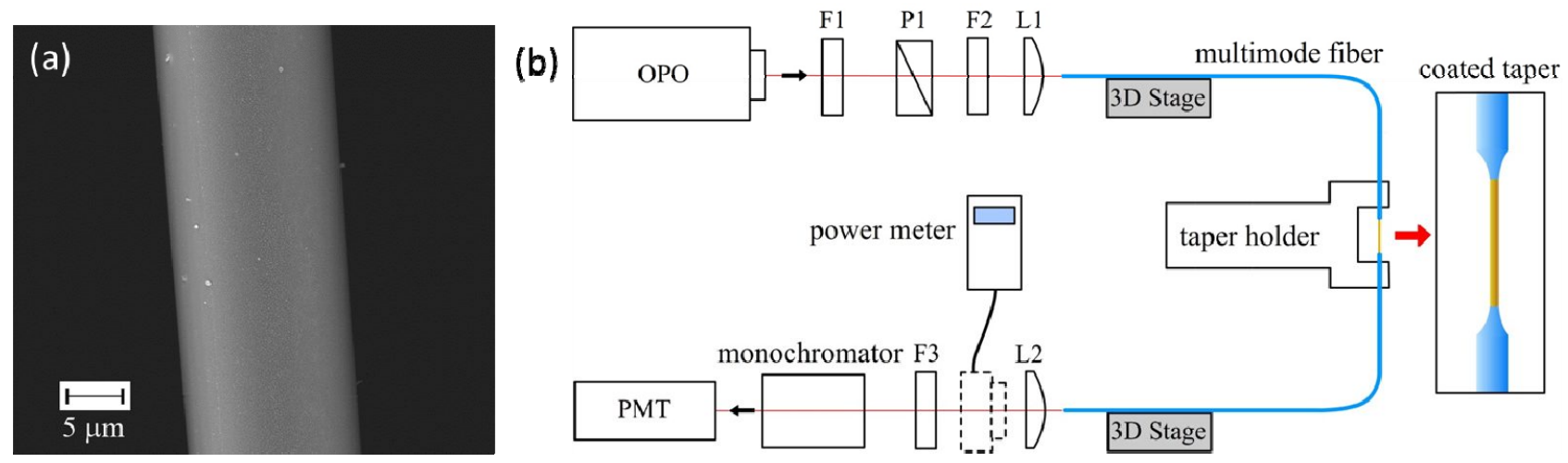

Fig. 1. (a) A SEM image of a nonlinear fiber taper coated with multiple layers of PAH/PB. (b) Schematic diagram of the experimental setup. An infrared, nanosecond-pulsed pump beam from an OPO is coupled into a multimode fiber. The beam propagates through a nonlinear taper, and interacts with the second-order nonlinear molecules on the taper surface. The output SHG signal is collimated, filtered and finally detected by a PMT.

The nonlinear optical properties of the fiber taper can be characterized using SHG. As shown in Fig. 1(b), we used an OPO to generate linearly polarized pump pulses (10 ns duration and $20 \mathrm{~Hz}$ repetition rate) at $1294 \mathrm{~nm}$. The pump pulse energy was adjusted by rotating the polarizer (P1). Two long-pass filters (F1 and F2) were used to remove background 
second harmonic signals generated by the polarizer and other optical components. The pump light was coupled into the nonlinear taper and generated second harmonic signals. At the fiber output, both the fundamental pump and the second harmonic signal were collimated back into free space, where we used a short pass filter (F3) to remove the pump light. The wavelength of the second harmonic signal is measured using a monochromator, and its power is detected by a photomultiplier tube (PMT).

To characterize SHG within the nonlinear fiber, we measured the dependence of SHG power on pump pulse energy. To eliminate variations due to different taper geometries, all nonlinear fiber samples were fabricated from the same bare silica taper. To achieve this goal, we first measured SHG produced by the bare silica taper. Then, we deposited 10 bilayers of PAH/PB film on the bare taper and performed SHG measurement again. This cycle (i.e., 10-bilayer film deposition followed by SHG measurement) was repeated until we reached 40 bilayers of PAH/PB film. As shown in Fig. 2 , we notice that second harmonic power increases quadratically as a function of the pump pulse energy, a characteristic feature of SHG. Furthermore, an increase in nonlinear film thickness directly leads to an increase in second harmonic power. For a 40-bilayer coating, we observe a 400 -fold increase in SHG power compared to the bare taper case. These results confirm that the measured second harmonic component is indeed generated by the nonlinear PAH/PB coating.

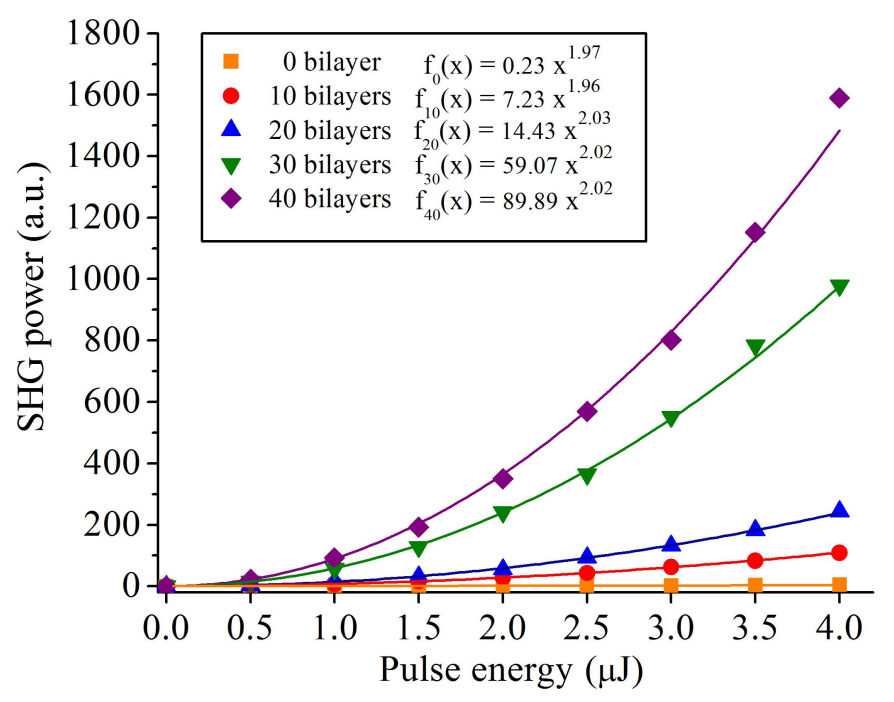

Fig. 2. SHG power as a function of pump pulse energy. The measurement was taken for a 3.8 - $\mu$ m-radius taper with 0 , 10 , 20,30 and 40 bilayers of nonlinear film. Each data point is the result of an average of over 100 measurement data and the error bar is smaller than the symbol. The lines are quadratic fits to the data.

For the silica taper with a uniform coating of PAH/PB molecules, the main limiting factor for significant SHG is how to achieve phase matching or QPM. Let us first consider the impact of phase mismatch. Our analysis is based on the following coupled mode equation ${ }^{10}$ :

$$
\frac{d E_{2 \omega}}{d z}=-i \frac{\omega E_{\omega}^{2}}{\varepsilon_{0} v_{g}} \frac{\delta}{\sqrt{\pi} \varepsilon_{r}^{3 / 2} a(z)} \chi_{z z z}^{(2)} \exp [-i \Delta \beta(z) z]
$$

where $\mathrm{E} \omega$ and $\mathrm{E}_{2 \omega}$ are respectively the electric field amplitude of the pump and SHG beams, $a(z)$ is taper radius, $\delta$ is nonlinear film thickness, $\chi_{z z z}^{(2)}$ is the zzz component of the second-order nonlinear susceptibility of the nonlinear film, and $v_{\mathrm{g}}$ is group velocity at $2 \omega$. (We choose the propagation distance $\mathrm{z}$ such that the narrowest fiber taper radius is at $\mathrm{z}=$ 0 .) In Eq. (1), the only parameter that cannot be readily obtained from experimental conditions is the phase-mismatch term $\Delta \beta(z)$. To calculate this term we assume the fundamental waves are in the $\mathrm{HE}_{11}$ mode. This assumption is based on the observation that fiber taper transition is gradual and smooth. We can use Eq. (1) to theoretically estimate the efficiency of second harmonic generation. For linearly polarized pump light, the second harmonic mode can be either $\mathrm{HE}_{21}$ or $\mathrm{TM}_{01}$ mode. For our present study, since the propagation constants of the $\mathrm{HE}_{21}$ and $\mathrm{TM}_{01}$ mode are very close, whether we choose $\mathrm{HE}_{21}$ or $\mathrm{TM}_{01}$ as the second harmonic mode should not make any significant differences. 
In order to use Eq. (1) to estimate second harmonic power, we need to know the taper radius profile. To experimentally obtain this parameter, we used an optical microscope to obtain an image of the entire fiber taper region, from which we extracted fiber radius $a$ at any given location and numerically calculated the value of $\Delta \beta(z)$ as a function of propagation distance z. (A representative example of taper image and extracted taper profile is shown in Fig. 3(a).) Once the $\Delta \beta(\mathrm{z})$ is known, we can then numerically integrate Eq. (1) and obtain the power of second harmonic wave. In Fig. 3(b), we use the $a(z)$ given in Fig. 3(a) and perform numerical integration starting from $\mathrm{z}=-\mathrm{L} / 2$ to $\mathrm{z}=\mathrm{L} / 2$ with various values of interaction length $\mathrm{L}$, and plot the second harmonic power as a function of the interaction length $\mathrm{L}$ in Fig. 3(b). We note that SHG power in Fig. 3(b) converges to a finite result for $\mathrm{L}$ greater than $3000 \mu \mathrm{m}$. Therefore, we consider $\mathrm{L}=3000$ $\mu \mathrm{m}$ to be the effective interaction length that defines the region of strong interaction between the nonlinear molecules and the pump field. This observation is also consistent with the taper profile shown in Fig. 3(a), where, at $\mathrm{z}= \pm 1500 \mu \mathrm{m}$, taper diameter grows to approximately twice of its waist value. The simulation result in Fig. 3(b) is consistent with experimental data: For a peak pump power of $400 \mathrm{~W}$ at $1294 \mathrm{~nm}$, the numerical integration indicates that we should expect a SHG power of $169.7 \mu \mathrm{W}$, whereas the experimentally measured second harmonic peak power is $49.8 \mu \mathrm{W}$. The agreement is reasonable, especially considering the fact that the numerical estimate is obtained without using any empirical fitting parameters.
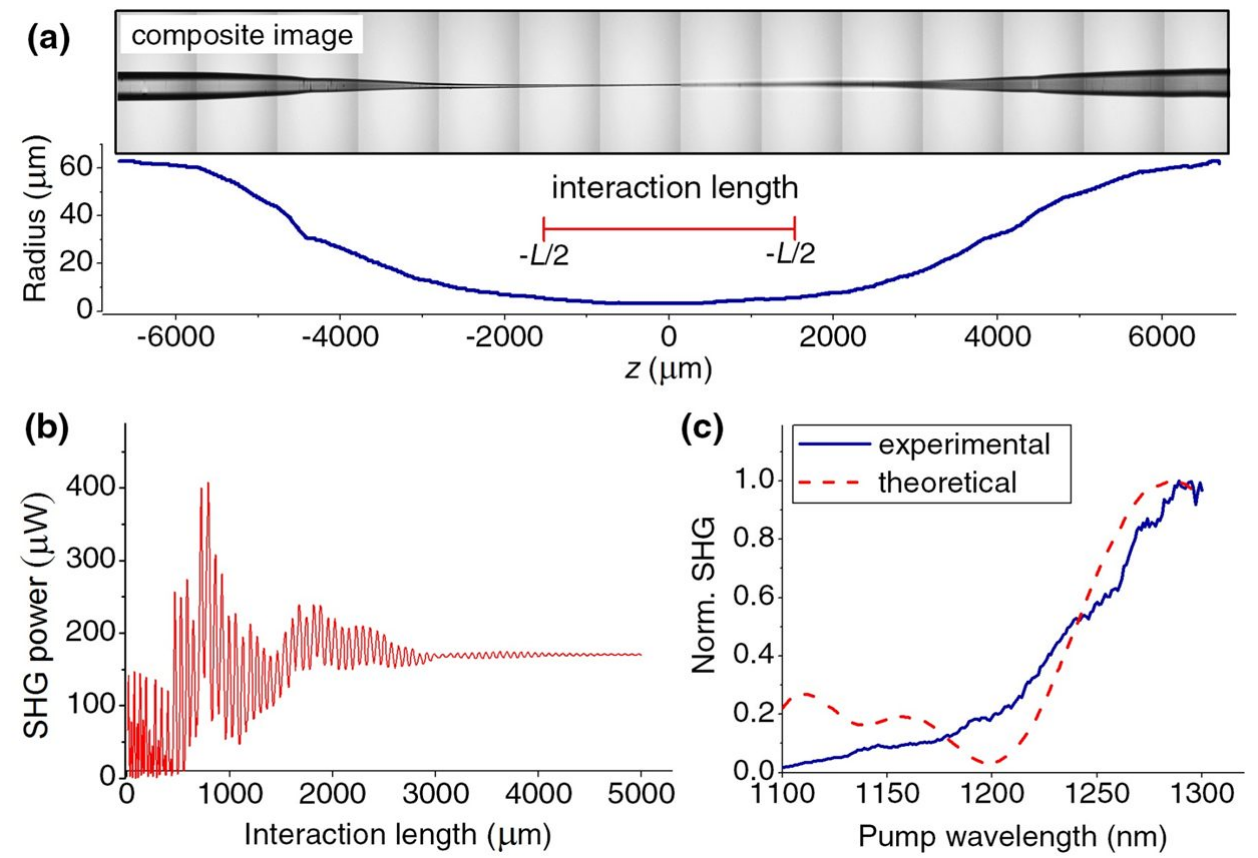

Fig. 3. (a) The profile of a 3.8- $\mu$ m taper obtained by combining sequential images taken using an optical microscope (Leica DMI-6000 B). (b) Theoretical estimate of SHG power as a function of taper interaction length. The result is obtained by numerically integrating Eq. (1) while using the taper profile determined in (a). (c) Theoretical simulation and experimental result of SHG power versus pump wavelength. Both results are normalized with respect to their corresponding peak SHG power.

By following the procedure outlined above and using parameters extracted from the actual nonlinear fiber, we can also calculate the strength of second harmonic signal generated by the nonlinear fiber as a function of pump wavelength. The numerical result is shown in Fig. 3(c) as a dashed line. The corresponding experimental result was obtained by tuning the OPO pump wavelength from $1100 \mathrm{~nm}$ to $1300 \mathrm{~nm}$ with a wavelength step of $1 \mathrm{~nm}$. The pump peak power was maintained at $400 \mathrm{~W}$ throughout the wavelength scan, and we used the monochromator and the PMT in Fig. 1(b) to measure SHG power. The experimentally measured SHG power is shown in Fig. 3(c) as a solid line. Again, we observe good agreement between the theoretical prediction and the experimental results.

The nonlinear fibers we have considered so far are not phase matched for SHG. As a result, the corresponding nonlinear conversion efficiency is extremely small. One possible solution is to create spatially periodic second-order nonlinearity that satisfies the condition for QPM. Here we present a UV-ablation-based approach that can address this challenge. In our approach, we expose the polymer-coated fiber taper sample to the focused laser beam produced by an Excimer laser 
(MPB Technology Inc.). The laser wavelength is $248 \mathrm{~nm}$, pulse width is $10 \mathrm{~ns}$, beam size is $\sim 1 \mathrm{~cm}^{2}$, and repetition rate is $5 \mathrm{~Hz}$. We used a mask with multiple metallic stripes with grating pitch $\Lambda=50 \mu \mathrm{m}$ to generate spatially periodic UV laser patterns. The fiber taper was placed under the mask and exposed to the UV laser for 5 minutes. Within the "shadowed" section (i.e., underneath the metal strips and not exposed to the UV laser), the assembled polymer layers remained the same. On the other hand, for the sections exposed to the UV laser, the assembled polymer layers were completely removed due to UV ablation. To verify the results of laser ablation, we deposited 20 bilayers of PAH/PSS polymers on a fiber taper. Then, we applied the aforementioned ablation technique to create spatially periodic patterns on the fiber taper. Since the PAH is conjugated with a red dye (Texas Red), the patterned polymer coating can be directly visualized using a fluorescence microscope, as shown in Fig. 4(a).

(a)

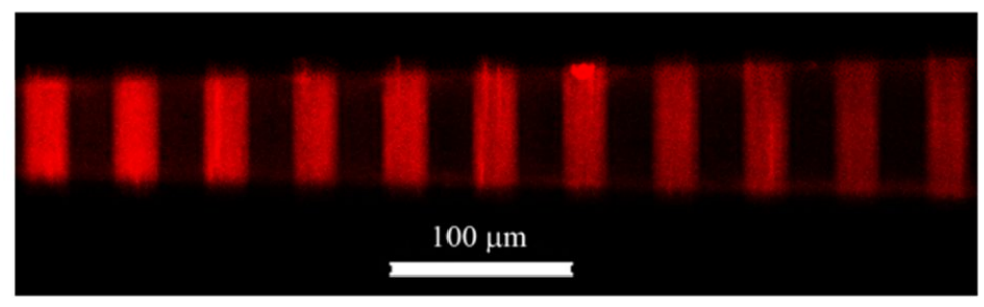

(b)

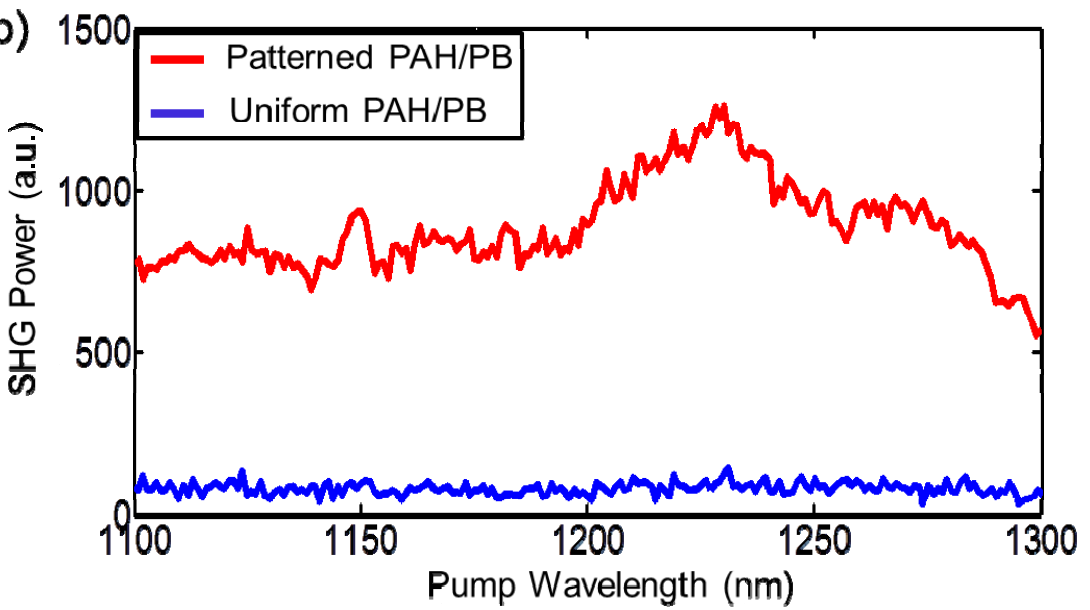

Fig. 4. (a) A fluorescence microscope image of a fiber taper coated PAH/PSS polymers, where PAH is conjugated with Texas Red and appears red under the fluorescence microscope. Part of the polymer coating is removed through UV ablation, which generates the spatially periodic pattern. (b) The SHG power generated by the nonlinear fiber taper before and after UV ablation based patterning. The significant enhancement of SHG power can be attributed to QPM.

For a preliminary test of QPM, we deposited 10 bilayers of PAH/PB on a fiber taper (waist radius of $\sim 3.75 \mu \mathrm{m}$ ). Afterwards, we used the UV ablation technique to generate spatially periodic patterns on the nonlinear fiber taper. We then measured the second harmonic signals generated by this nonlinear fiber taper sample before and after UV ablation patterning. The results, as seen in Fig. 4(b), clearly show enhanced SHG for the patterned sample, which suggests that some degree of QPM has been realized in the patterned nonlinear fiber sample.

\section{FLUORESCENCE LIFETIME BASED CHARACTERIZATION OF A TUNABLE PLASMONIC SYSTEM}

In this section, we present s study of a tunable plasmonic system based on Purcell effects and fluorescence lifetime measurements ${ }^{17}$. As pointed out by Purcell, an optical micro- or nano-structure can have a dramatic impact on the process of spontaneous emission in its vicinity ${ }^{18}$. More precisely, a fluorophore's spontaneous emission rate is controlled by vacuum field fluctuations and is proportional to the photonic density of states (PDOS). For a plasmonic system, the PDOS has extremely sensitive dependence on the relative position between the plasmonic nanostructure and the 
fluorophore ${ }^{19}$. Therefore, it is possible to establish a one-to-one correspondence between fluorescence lifetime, PDOS, and fluorophore position. We can then use this relationship to characterize a tunable plasmonic system composed of swellable polymers and plasmonic nanostructures.

Our first task is to establish a quantitative link between fluorescence lifetime, PDOS, and fluorophore position. We mainly consider a configuration in which a layer of self-assembled polymer films separates a plasmonic substrate and fluorophores, as illustrated in Fig. 5(a). A key observation of our work is that we can vary the distance between the fluorophore and the plasmonic substrate by either changing the number of polymer monolayers (in the case of fixed structures) or by swelling / deswelling the polymer films (in the case of tunable structures).
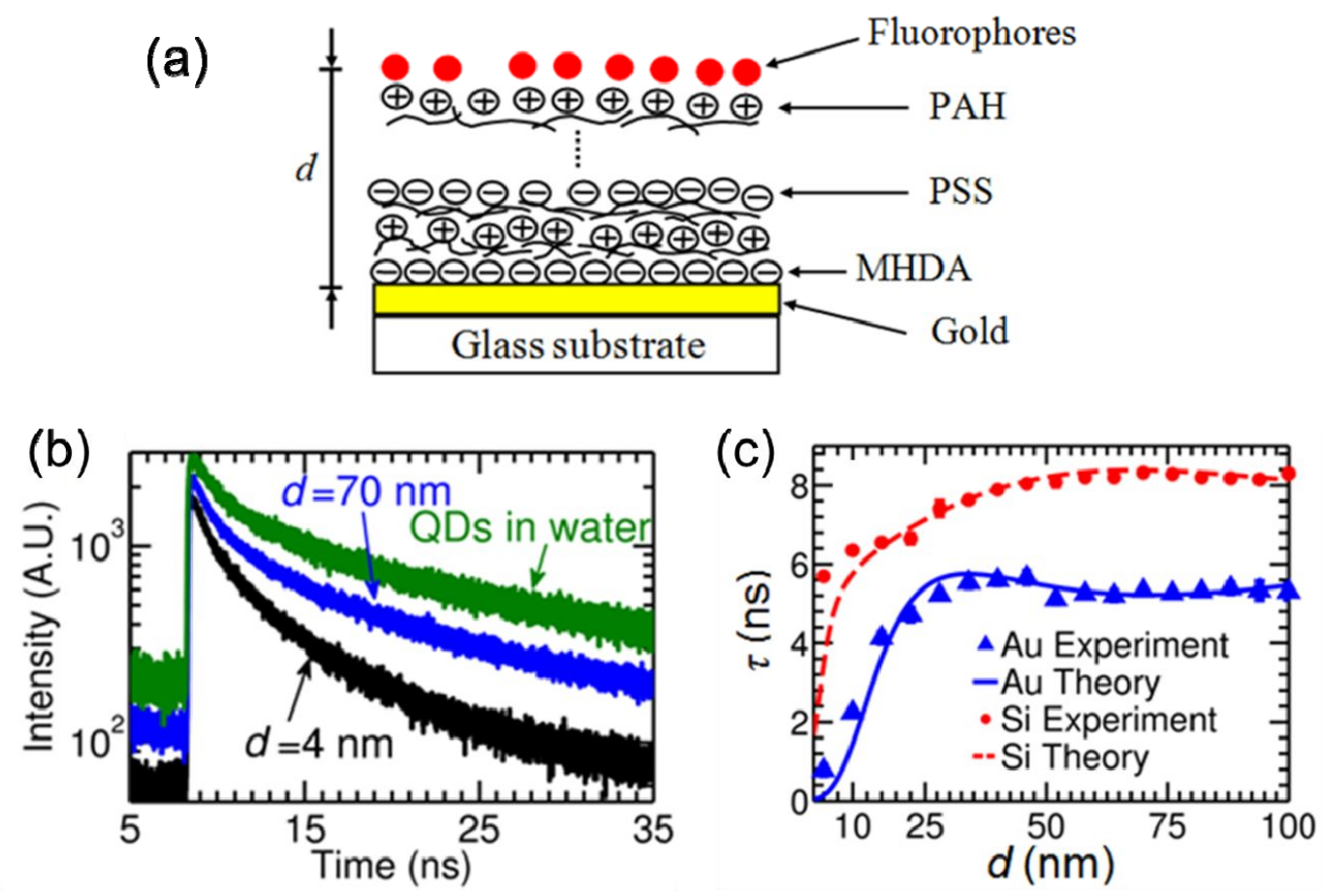

Fig. 5. (a) Schematic of the plasmonic structure. The PAH/PSS polymer layers (with thickness d) serve as a "spacer" that separates the fluorophores and the planar Au substrate. (b) The photoluminescence of samples with different spacer layer thickness d. (c) The average fluorescence lifetime as a function of the distance between the fluorophores and the planar substrate.

To fabricate the structure illustrated in Fig. 5(a), we first immersed an Au coated glass slide in a $1 \mathrm{mM}$ solution of mercaptohexadecanoic acid (MHDA) in ethanol ( $\mathrm{pH} \sim 2$ ) for 24 hours to create a uniformly negatively charged surface. We then fabricated an LbL film consisting of alternating layers of PAH and PSS monolayers on the substrate following Ref. [20]. After obtaining the desired number of layers capped with a positively charged PAH layer, we immersed the sample in a suspension of negatively charged fluorophores, which adsorbed onto the film surface due to electrostatic interaction. On $\mathrm{Si}$, the thiol layer is not required, and $\mathrm{LbL}$ films were assembled directly on a cleaned substrate. By adjusting the $\mathrm{pH}$ values of the solutions used for LbL assembly, the PAH/PSS polymer layers can be made to be either fixed or swellable.

The fluorescence lifetime of the samples was measured using a time-correlated single-photon counting (TCSPC) (Picoharp 300, PicoQuant) and a pulsed $473 \mathrm{~nm}$ laser diode (BDL-473-C, Becker and Hickl) as the excitation source. Experimentally, we found that QD fluorescence was multiple exponential, as shown in Fig. 5(b). In order to consistently evaluate fluorescence dynamics, we therefore define the average fluorescence lifetime $\tau$ as $\tau=\int_{0}^{\infty} t I(t) d t / \int_{0}^{\infty} I(t) d t$, where $\mathrm{I}(\mathrm{t})$ is the experimentally measured fluorescence intensity.

The principle for fluorescence-lifetime-based thickness measurement is based on Purcell effects. Specifically, in the vicinity of the plasmonic film, the average lifetime depends on the intrinsic fluorescence lifetime $\tau_{0}$, the quantum yield q, and the modified PDOS, and can be written as ${ }^{17}$ : 


$$
\tau=\frac{\tau_{0}}{1-q[1-\gamma(d)]}
$$

where the quantum yield $\mathrm{q}$ is the ratio between the fluorescence decay rate and the total decay rate, $\tau_{0}$ is the intrinsic fluorescence lifetime, and d denotes fluorophore location. In Eq. (2), the parameter $\gamma$ accounts for changes in PDOS and is defined as $\gamma(d)=\rho(d) / \rho_{0}(d)$, where $\rho(d)$ and $\rho_{0}(d)$ represents the PDOS in the presence and the absence of the Au substrate, respectively. Eq. (2) clearly demonstrates that the fluorescence lifetime can be dramatically changed by the PDOS at the fluorophore location. In Fig. 5(c), we show the experimentally measured QD fluorescence lifetime as a function of the polymer spacer thickness d. The dramatic change in fluorescence lifetime near the Au substrate is obvious and can be attributed to the existence of plasmonic resonance.

For a planar substrate, the value of $\gamma$ can be analytically calculated using the formula in Ref. [21]. It should be emphasized that $\gamma$ is essentially the Purcell factor and that its value depends only on the wavelength, the dielectric constants of the surrounding media, and the fluorophore location. The value of $\gamma$, however, does not depend on specific fluorophores. For the planar Au substrate we investigated, the theoretically predicted $\gamma$ values are shown in Fig. 6(a). Using this theoretical curve and setting $\mathrm{q}=35 \%$ and $\tau_{0}=7 \mathrm{~ns}$, we can treat Eq. (1) as a look-up table and directly link the measured fluorescence lifetime $\tau$ with fluorophore position $\mathrm{d}$. Since the QDs are located at the top of the LbL film, the value of $d$ is essentially the sum of LbL film thickness and QD radius. In Fig. 6(b), we show the experimentally measured QD photoluminescence produced by four samples with different LbL film thickness. In Fig. 6(c), we show film thicknesses extrapolated using Eq. (1) (i.e., $d_{\mathrm{PDOS}}$ ) versus the values obtained using ellipsometry (i.e., $d_{\text {Ellip }}$ ). Note that in our Purcell-factor-based approach, there is no free fitting parameter, where all values are obtained experimentally. The good agreement in Fig. 6(c) clearly suggests that our approach can measure LbL film thickness with nanoscale resolution.
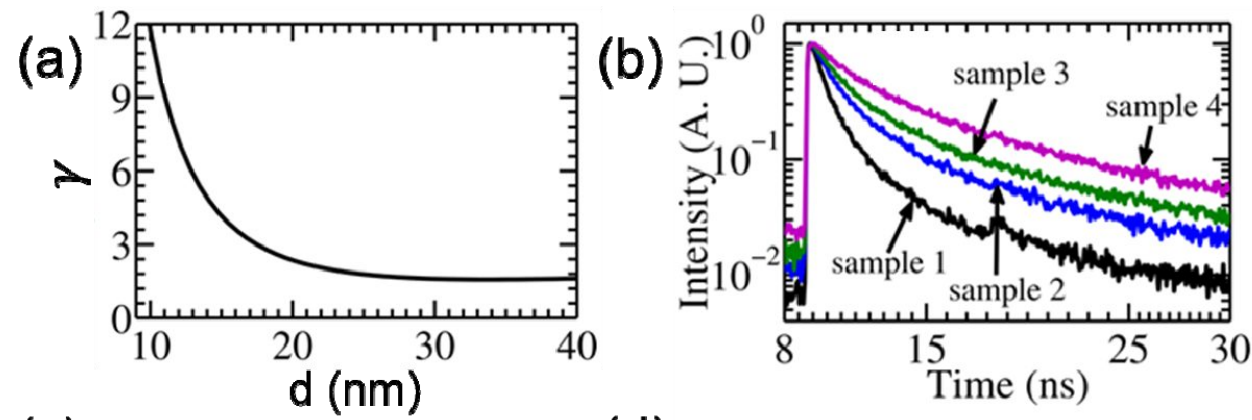

(c)

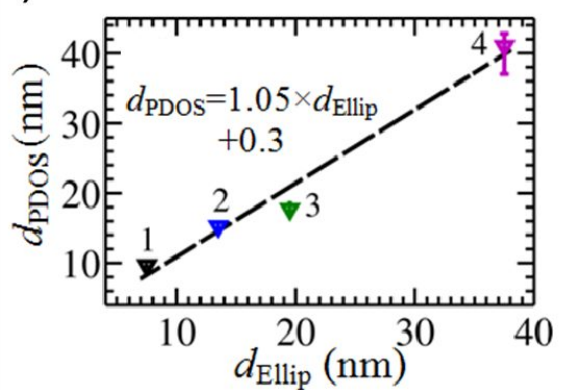

(d)

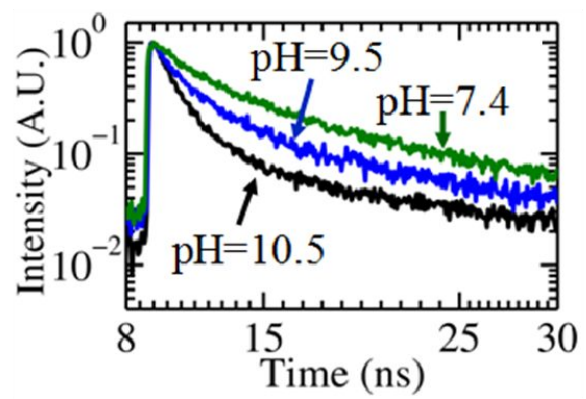

Fig. 6. (a) The modification of PDOS as a function of the spacer layer thickness d. (b) The photoluminescence of four samples with different spacer layer thickness. (c) For the four samples in (b), we can extract their polymer layer thickness from fluorescence lifetime measurements. These results $\left(\mathrm{d}_{\mathrm{PDOS}}\right)$ agree well with layer thickness obtained from ellipsometry measurements $\left(\mathrm{d}_{\text {Ellip }}\right)$. (d) The photoluminescence of a tunable plasmonic structure placed in solutions with different $\mathrm{pH}$ values. The significant modifications in fluorescence lifetime indicate dramatic changes in layer thickness.

We can use QD fluorescence lifetime to characterize the tunable plasmonic system illustrated in Fig. 5(a). To achieve tunability, we set the $\mathrm{pH}$ values of all solutions used in the LbL assembly processes to be 9.3 to ensure significant polymer swelling and deswelling. Afterwards, we immersed the sample in aqueous solutions with specific $\mathrm{pH}$ values for 
10 minutes and then measured QD fluorescence using the TCSPC system. Fig. 6(d) shows three examples of QD photoluminescence produced by the same sample but obtained under three different $\mathrm{pH}$ values, 7.4, 9.5, and 10.5. Based on the average QD fluorescence lifetime, we find that the polymer spacer thickness can vary anywhere from $10 \mathrm{~nm}$ to 40 $\mathrm{nm}$, depending on the solution $\mathrm{pH}$ values and swelling / deswelling histories.

\section{CONCLUSIONS}

In summary, we report the fabrication and characterization of two functional optical systems based on self-assembled nanomaterials. In particular, we find that it is possible to produce significant second-order nonlinearity in a silica-based fiber structure by coating a silica fiber taper with multiple layers of $\mathrm{PAH} / \mathrm{PB}$ polymer coatings. We characterize such nonlinear fiber structures using SHG and find reasonable agreement between the experimentally measured second harmonic powers and the theoretically predicted values. We further develop a UV-ablation based approach for QPM and obtained significantly enhanced second harmonic signals in a nonlinear fiber taper with quasi-phase-matched PAH/PB coatings. Next, we investigate a tunable plasmonic system based on polymer swelling / deswelling and fluorescence lifetime measurements. We first confirmed that it is possible to measure the polymer layer thickness with nanoscale resolutions by linking QD fluorescence lifetime with the modification of PDOS through Purcell effects. We then use this method to characterize the swelling / deswelling of a PAH/PSS multilayer structure assembled over a planar Au substrate and confirmed that it is possible to modify the thickness of a plasmonic nanostructure by almost $400 \%$.

\section{REFERENCES}

[1] Decher, G., "Fuzzy nanoassemblies: toward layered polymeric multicomposites," Science, 277, 1232-1237 (1997).

[2] Itano, K., Choi, J., and Rubner, M. F., "Mechanism of the pH-Induced discontinuous swelling/deswelling transitions of Poly(allylamine hydrochloride)-containing polyelectrolyte multilayer films," Macromolecules. 38, 3450 (2005).

[3] Agrawal, G. P., [Nonlinear Fiber Optics], Elsevier, Singapore, (2007).

[4] Antonyuk, B. P., Novikova, N. N., Didenko, N. V., and Aktsipetrov, O. A., "All optical poling and second harmonic generation in glasses: theory and experiment," Phys. Lett. A. 287, 161-168 (2001).

[5] Balakirev, M. K., Smirnov, V. A., and Vostrikova, L. I., "Photorefractive effect on all optical polling of glass," J. Opt. A: Pure Appl. Opt. 5, S437-S443 (2003).

[6] Tombelaine, V., Buy-Lesvigne, C., Leproux, P., Couderc, V., and Melin, G., "Optical poling in germanium-doped microstructured optical fiber for visible supercontinuum generation,” Opt. Lett. 33, 2011-2013 (2008).

[7] Myers, R. A., Mukherjee, N., and Brueck, S. R. J., "Large second-order nonlinearity in poled fused silica fiber," Opt. Lett. 16, 1732-1734 (1991).

[8] An, H., and Fleming, S., "Second-order optical nonlinearity in thermally poled borosilicate glass," Appl. Phys. Lett. 89, 181111(1)-181111(3) (2006).

[9] Moura, A. L., de Araujo, M. T., Vermelho, M. V. D., and Aitchison, J. S., "Improved stability of the induced second-order nonlinearity in soft glass by thermal poling," J. Appl. Phys. 100, 033509(1)-033509(5) (2006).

[10] Xu, Y., Wang, A., Heflin, J. R., and Liu, Z., "Proposal and analysis of a silica fiber with large thermodynamically stable second order nonlinearity," Appl. Phys. Lett. 90, 211110(1)-211110(3) (2007).

[11] Xu, Y., Han, H., Wang, A., Liu, Z., and Heflin, J. R., "Second order parametric processes in nonlinear silica microspheres," Phys. Rev. Lett. 100, 163905(1)-163905(4) (2008).

[12] Daengngam, C., Hofmann, M. C., Liu, Z., Wang, A., Heflin, J. R., and Xu, Y., "Demonstration of a cylindrically symmetric second-order nonlinear fiber with self-assembled organic surface layers," Opt. Express, 19, 10326-10335 (2011).

[13]Heflin, J. R., Guzy, M. T., Neyman, P. J., Gaskins, K. J., Brands, C., Wang, Z., Gibson, H. W., Davis, R. M., and Van Cott, K. E., "Efficient, thermally stable, second order nonlinear optical response in organic hybrid covalent/ionic self-assembled films," Langmuir, 22, 5723-5727 (2006).

[14] Stuart, M. A. C., et al., "Emerging applications of stimuli-responsive polymer materials," Nat. Mater., 9, 101-113 (2010).

[15] Tokarev, I., Tokareva, I., and Minko, S., "Optical nanosensor platform operating in near-physiological pH range via polymer-brush-mediated plasmon coupling," ACS Applied Materials \& Interfaces, 3, 143-146, (2011).

[16] Brisk, T. A., and Li, Y. W., “The shape of fiber tapers,” J. Lightwave. Technol. 10, 432-438 (1992). 
[17] Ashry, I., Zhang, B., Stoianov, S. V., Daengngam, C., Heflin, J. R., Robinson, H. D., Xu, Y., "Probing the photonic density of states using layer-by-layer self-assembly,” Opt. Lett., 37, 1835-1837 (2012).

[18] Noda, S., Fujita, M., and Asano, T., "Spontaneous-emission control by photonic crystals and nanocavities," Nature Photonics, 1, 449 (2007).

[19] Vamsi. K. Komarala, Yury. P. Rakovich, A. L. Bardley, Stephen J. Byrne, Yurii K. Gun'ko, N. Gaponik and A. Eychmüller, "Off-resonance surface plasmon enhanced spontaneous emission from CdTe quantum dots," Applied Physics Letters. 89, 253118 (2006).

[20] Ray, K., Badugu, R., and Lakowicz, J. R., "Polyelectrolyte layer-by-layer assembly to control the distance between fluorophores and plasmonic nanostructures," Chemistry of Materials, 19, 5902 (2007).

[21] Chance, R. R., Prock, A., and Silbey, R., "Molecular fluorescence and energy transfer near interfaces," Advances in Chemical Physics, 37, 1 (1978). 